\title{
The ethnobotanical notes from Nizip (Gaziantep-Turkey)
}

\author{
Gizem Bulut*, Ali Korkmaz, Ertan Tuzlacı \\ Department of Pharmaceutical Botany, Faculty of Pharmacy, Marmara University, 34668, İstanbul, Turkey
}

Cite this article as: Bulut G, Korkmaz A, Tuzlacı E (2017). The ethnobotanical notes from Nizip (Gaziantep-Turkey). Istanbul J Pharm 47 (2): 57-62.

\begin{abstract}
This paper reports folk medicinal and food plants of Nizip (Gaziantep) located in the south part of Turkey. The purpose of the study is to gather, determine, and record the plants that are used as source of food and medicine by local people. The information was obtained from participants in face to face interviews; furthermore, the specimens of the plants were collected. Voucher specimens were deposited at the Herbarium of the Faculty of Pharmacy, Marmara University. Totally, twenty-seven plants are recorded as used as traditional folk medicine for the region, and thirteen of these are also used as a source of food. Among them, 20 taxa were wild and 7 taxa were cultivated plants. According to the majority of the plants which have similar usage, the plants are mostly used for gastrointestinal, respiratory system diseases and diabetes.
\end{abstract}

Keywords: Ethnobotany, folk medicinal plants, food plants, Nizip, Turkey

\section{INTRODUCTION}

Turkey has a rich traditional culture because the country is located in region where the effect of lots of civilization was observed. The important part of this culture include nutrition plants that can be eaten and folk medicine (Bulut, 2016). This riches is displayed with ethnobotany researches which is done in different regions of Turkey. Nizip where we performed preliminary researches take part in the east of Turkey but there isn't enough work in the region (Akan et al. 2008; Altundağ and Öztürk 2011; Bulut et al. 2016; Çakılcıoğlu and Türkoğlu 2010; Çakılcıoğlu et al. 2010, 2011; Doğan and Tuzlacı 2015; Kaval et al. 2014; Mükemre et al. 2015; Özgen et al. 2004; Özgökçe and Özçelik 2004; Sezik et al. 1997; Şığva and Seçmen 2009; Tabata et al. 1994; Tetik et al. 2013; Tuzlacı and Doğan 2010; Yeşil and Akalın 2009).

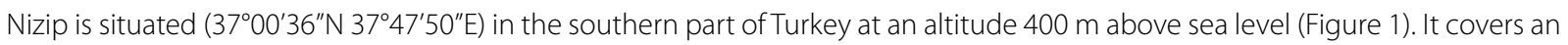
area of $1.031 \mathrm{~km}^{2}$ and its population is 109.285. Nizip is surrounded by Yavuzeli in the north, Karkamış in the south, Birecik (Şanlıurfa) in the east and Şehitkamil and Oğuzeli in the west. The main crops of Nizip are olive and pistachio. Also, Zeugma ancient city that is located $10 \mathrm{~km}$ from Nizip has a historical importance in the region.

\section{MATERIALS AND METHODS}

This ethnobotanical study addresses the use of wild plants as a source of food and medicine. The study was made in 2012 and its materials were the plants (27 taxa) collected during the field work. The information was obtained through open and semi-structured interviews from the local people (Alexiades 1996; Cotton 1996; Martin 1995) with local people. The interviews were made as general conversations with a strict questionnaire (Appendix 1). The information about the local names, the part(s) used, the ailments treated, the therapeutic effect, the preparation, the methods of administration, and the duration of treatment was recorded. The "Flora of Turkey and the East Aegean Islands" (Davis 1965-1985; Davis et al. 1988; Güner et al. 2000) were mainly used for the identification of the plants. The plant specimens are kept in the Herbarium of the Faculty of Pharmacy, Marmara University (MARE). 
Table 1. Medicinal and food plants of Nizip (Gaziantep-Turkey)

\begin{tabular}{|c|c|c|c|c|c|c|c|}
\hline $\begin{array}{l}\text { Botanical name, } \\
\text { Family and } \\
\text { Specimen number }\end{array}$ & Local name & $\begin{array}{l}\text { Plant part } \\
\text { used } \\
\text { (medicine) T }\end{array}$ & $\begin{array}{c}\text { Ailments } \\
\text { treated/ } \\
\text { Therapeutic effect }\end{array}$ & $\begin{array}{c}\text { Preparation } \\
\text { and } \\
\text { t Administration }\end{array}$ & $\begin{array}{l}\text { Plant part } \\
\text { used (food) }\end{array}$ & $\begin{array}{l}\text { Modes of } \\
\text { consumption }\end{array}$ & References \\
\hline $\begin{array}{l}\text { Alcea dissecta (Baker) } \\
\text { Zohary } \\
\text { (Malvaceae, MARE 15333) }\end{array}$ & Hatmi çiçeği & $\begin{array}{l}\text { Flowers } \\
\text { Flowers }\end{array}$ & $\begin{array}{l}\text { Cough } \\
\text { Gastro-intestinal } \\
\text { disorders }\end{array}$ & $\begin{array}{l}\text { Infusion, int. } \\
\text { Infusion, int. }\end{array}$ & & & $(15,2)^{b}$ \\
\hline $\begin{array}{l}\text { Astragalus lamarckii Boiss. } \\
\text { [Astracantha lamarckii } \\
\text { (Boiss.) Podl.] } \\
\text { (Fabaceae, MARE 15337) }\end{array}$ & Geven & Aerial parts & Cold & Infusion, int. & & & \\
\hline $\begin{array}{l}\text { Capparis spinosa } L . \\
\text { (Capparaceae, MARE } \\
\text { 15329) }\end{array}$ & $\begin{array}{l}\text { Kember } \\
\text { Flo }\end{array}$ & $\begin{array}{l}\text { Gemmae } \\
\text { wering branches }\end{array}$ & $\begin{array}{l}\text { Rheumatism } \\
s \text { Expectorant }\end{array}$ & $\begin{array}{l}\text {-, eaten } \\
\text { Decoction, int. }\end{array}$ & & & $(2)^{\mathrm{b}}$ \\
\hline $\begin{array}{l}\text { a Cupressus sempervirens L } \\
\text { (Cupressaceae, } \\
\text { MARE15345) }\end{array}$ & L. Çam Le & $\begin{array}{l}\text { aves \& branches } \\
\text { Cones }\end{array}$ & $\begin{array}{l}\text { Constipation } \\
\text { Enuresis nocturna }\end{array}$ & $\begin{array}{l}\text { Decoction, int. } \\
\text { Decoction, int. }\end{array}$ & & & \\
\hline $\begin{array}{l}\text { Ficus carica L. subsp. carica } \\
\text { (Moraceae, MARE 15356) }\end{array}$ & ta İncir & $\begin{array}{l}\text { Latex } \\
\text { Dried fruits }\end{array}$ & $\begin{array}{c}\text { Wart } \\
\text { Constipation }\end{array}$ & $\begin{array}{l}- \text {, ext. } \\
- \text {, ext. }\end{array}$ & Fruits & Eaten raw, jam & $(2)^{\mathrm{b}}$ \\
\hline $\begin{array}{l}\text { a Hibiscus esculentus L. } \\
\text { [Abelmoschus esculentus } \\
\text { (L.) Moench] } \\
\text { (Malvaceae, MARE 15353) }\end{array}$ & Bami & $\begin{array}{l}\text { Flowers } \\
\text { Flowers }\end{array}$ & $\begin{array}{l}\text { Constipation } \\
\text { Gastro-intestinal }\end{array}$ & $\begin{array}{l}\text { Decoction, int. } \\
\text { Decoction, int. } \\
\text { disorders }\end{array}$ & Fruits & Cooked & \\
\hline $\begin{array}{l}\text { a Juglans regia L. } \\
\text { (Juglandaceae, } \\
\text { MARE15351) }\end{array}$ & Ceviz & $\begin{array}{l}\text { Leaves } \\
\text { Seed }\end{array}$ & $\begin{array}{l}\text { Diabetes } \\
\text { Dysmnesia }\end{array}$ & $\begin{array}{l}\text { Decoction, int. } \\
\quad-\text {, eaten }\end{array}$ & Seed & Eaten raw & $\begin{array}{c}\text { Diabetes }(14) \\
(2,6,8,10,11 \\
13,15,16)^{b}\end{array}$ \\
\hline $\begin{array}{l}\text { Lactuca serriola L. } \\
\text { (Asteraceae, } \\
\text { MARE 15338) }\end{array}$ & Sirok & $\begin{array}{l}\text { Aerial parts } \\
\text { Aerial parts } \\
\text { Aerial parts } \\
\text { Aerial parts }\end{array}$ & $\begin{array}{l}\text { Liver diseases } \\
\text { Kidney ailments } \\
\text { Digestive } \\
\text { Expectorant }\end{array}$ & $\begin{array}{l}\text { Decoction, int. } \\
\text { Decoction, int. } \\
\text { Decoction, int. } \\
\text { Decoction, int. }\end{array}$ & & & $(4,12,14,)^{b}$ \\
\hline $\begin{array}{l}\text { Mentha longifolia (L.) } \\
\text { Hudson subsp. typhoides } \\
\text { (Brig.) Harley var. } \\
\text { typhoides (Lamiaceae, } \\
\text { MARE 15335) }\end{array}$ & Punk & $\begin{array}{l}\text { Aerial parts } \\
\text { Aerial parts } \\
\text { Aerial parts }\end{array}$ & $\begin{array}{c}\text { Cold } \\
\text { Appetizer } \\
\text { Gastrointestinal } \\
\text { disorders }\end{array}$ & $\begin{array}{l}\text { Infusion, int. } \\
\text { Infusion, int. } \\
\text { Infusion, int. }\end{array}$ & Aerial parts & Spice & $\begin{array}{c}\text { Cold }(2,10,13) \\
\text { Gastrointestinal } \\
\text { system } \\
\text { diseases }(16,11) \\
(3,4,5,6,8,15)^{b} \\
\text { Spice }(3,9)\end{array}$ \\
\hline $\begin{array}{l}\text { a Olea europaea L. } \\
\text { var. europaea } \\
\text { (Oleaceae, } \\
\text { MARE 15336) }\end{array}$ & Zeytin & $\begin{array}{l}\text { Leaves } \\
\text { Leaves } \\
\text { Leaves } \\
\text { Fruits }\end{array}$ & $\begin{array}{l}\text { Appetizer } \\
\text { Diabetes } \\
\text { High cholesterol } \\
\text { Constipation }\end{array}$ & $\begin{array}{l}\text { Decoction, int. } \\
\text { Decoction, int. } \\
\text { Decoction, int. } \\
\text { Olive oil, eaten }\end{array}$ & $\begin{array}{l}\text { Fruits } \\
\text { Fruits }\end{array}$ & $\begin{array}{c}\text { Eaten raw } \\
\text { Pressed into oil }\end{array}$ & $(11,12)^{\mathrm{b}}$ \\
\hline $\begin{array}{l}\text { Pistacia khinjuk Stocks } \\
\text { (Anacardiaceae, MARE } \\
\text { 15352) }\end{array}$ & Menengic & Fruits & Cold & $\begin{array}{l}\text { Ground and make a } \\
\text { coffee, int. }\end{array}$ & & & $(1)^{\mathrm{b}}$ \\
\hline $\begin{array}{l}\text { a Pistacia vera L. } \\
\text { (Anacardiaceae, MARE } \\
\text { 15346) }\end{array}$ & Antep fıstığı & $\begin{array}{l}\text { Fruit } \\
\text { Resina }\end{array}$ & $\begin{array}{l}\text { Diabetes } \\
\text { Stomach ulcer }\end{array}$ & $\begin{array}{l}\text {-, eaten } \\
\text {-, int. }\end{array}$ & Seed & Eaten raw & $\begin{array}{l}\text { (12)b1 } \\
\text { Eaten raw (12) }\end{array}$ \\
\hline $\begin{array}{l}\text { Platanus orientalis L. } \\
\text { (Platanaceae, MARE } \\
\text { 15355) }\end{array}$ & Çınar & $\begin{array}{l}\text { Leaves } \\
\text { Leaves } \\
\text { Leaves }\end{array}$ & $\begin{array}{l}\text { Calcification } \\
\text { Antipyretic } \\
\text { Toothache }\end{array}$ & $\begin{array}{l}\text { Decoction, int. } \\
\text { Decoction, int. } \\
\text { Decoction, gargle }\end{array}$ & & & $(12,14)^{b}$ \\
\hline $\begin{array}{l}\text { Portulaca oleracea L. } \\
\text { (Portulacaceae, MARE } \\
\text { 15332) }\end{array}$ & Pirpirin & Aerial parts & $\begin{array}{l}\text { Gastrointestinal } \\
\text { disorders }\end{array}$ & Decoction, int. A & $\begin{array}{l}\text { Aerial parts } \\
\text { Aerial parts }\end{array}$ & $\begin{array}{l}\text { Boiled then } \\
\text { salad (+ yogurth) } \\
\text { Salad }\end{array}$ & $\begin{array}{c}\text { Gastro-intestinal } \\
\text { disorders (14) } \\
(2,3,5)^{\mathrm{b}} \\
\text { Salad }(3,12)\end{array}$ \\
\hline $\begin{array}{l}\text { Punica granatum L. } \\
\text { (Punicaceae, } \\
\text { MARE 15348) }\end{array}$ & Nar ağacı & $\begin{array}{l}\text { Seed } \\
\text { Seed } \\
\text { Seed }\end{array}$ & $\begin{array}{l}\text { Constipation } \\
\quad \text { Cold } \\
\text { Anthelmintic }\end{array}$ & $\begin{array}{l}\text {-, eaten } \\
\text {-, eaten } \\
\text {-, eaten }\end{array}$ & $\begin{array}{l}\text { Seed } \\
\text { Seed } \\
\end{array}$ & $\begin{array}{c}\text { Eaten raw } \\
\text { Squeezed } \\
\text { [“nar ekșisi”) } \\
\text { then added in salad }\end{array}$ & \\
\hline
\end{tabular}




\section{Table 1. Medicinal and food plants of Nizip (Gaziantep-Turkey)}

\begin{tabular}{|c|c|c|c|c|c|c|c|}
\hline $\begin{array}{l}\text { Botanical name, } \\
\text { Family and } \\
\text { Specimen number }\end{array}$ & Local name & $\begin{array}{c}\text { Plant part } \\
\text { used } \\
\text { (medicine) }\end{array}$ & $\begin{array}{c}\text { Ailments } \\
\text { treated/ } \\
\text { Therapeutic effect }\end{array}$ & $\begin{array}{l}\text { Preparation } \\
\text { and } \\
\text { Administration }\end{array}$ & $\begin{array}{l}\text { Plant part } \\
\text { used (food) }\end{array}$ & $\begin{array}{l}\text { Modes of } \\
\text { consumption }\end{array}$ & References \\
\hline $\begin{array}{l}\text { Quercus brantii Lindley } \\
\text { (Fagaceae, MARE 15344) }\end{array}$ & $\begin{array}{l}\text { Palamut ağac } \\
\text { Mature fruits } \\
\text { Mature fruits }\end{array}$ & $\begin{array}{ll}\text { acl } & \text { Leaves } \\
\text { ts } & \text { Diabetes } \\
\text { ts } & \text { Diarrhea }\end{array}$ & $\begin{array}{l}\text { Itching } \\
\text { Crushed, int. } \\
\text { Crushed, int. }\end{array}$ & Decoction, ext. & Fruit & Roasted & Diabetes (15) \\
\hline $\begin{array}{l}\text { Rhus coriaria L. } \\
\text { (Anacardiaceae, } \\
\text { MARE 15342) }\end{array}$ & \multicolumn{2}{|c|}{$\begin{array}{c}\text { Sumak Leaves \& branches } \\
\text { Fruits }\end{array}$} & $\begin{array}{l}\text { Diabetes } \\
\text { Appetizer }\end{array}$ & $\begin{array}{l}\text { Decoction, int. } \\
\text { Decoction, int. }\end{array}$ & Fruit & $\begin{array}{l}\text { Crushed then } \\
\text { added in salad }\end{array}$ & $(2,6,13)^{\mathrm{b}}$ \\
\hline $\begin{array}{l}\text { Rubus sanctus Schreber } \\
\text { (Rosaceae, MARE 15347) }\end{array}$ & Böğürtlen & $\begin{array}{l}\text { Fruits } \\
\text { Fruits }\end{array}$ & $\begin{array}{c}\text { Cough } \\
\text { Respiratory system } \\
\text { diseases }\end{array}$ & $\begin{array}{l}\text { Jam, eaten } \\
\text { Jam, eaten }\end{array}$ & Fruit & Eaten & $\begin{array}{l}\text { Respiratory } \\
\text { system } \\
\text { diseases (14) } \\
(2,5,6,12)^{b}\end{array}$ \\
\hline $\begin{array}{l}\text { Salix acmophylla Boiss. } \\
\text { (Salicaceae, MARE 15341) }\end{array}$ & Biy ağacı & Leaves & Rhematism & Decoction, int. & & & \\
\hline $\begin{array}{l}\text { Solanum nigrum } \\
\text { L. subsp. schultesii (Opiz) } \\
\text { Wessely } \\
\text { (Solanum decipiens Opiz) } \\
\text { (Solanaceae, MARE 15343) }\end{array}$ & $\begin{array}{l}\text { Köpek } \\
\text { domatesi }\end{array}$ & Aerial parts & $\begin{array}{c}\text { Eye } \\
\text { diseases }\end{array}$ & $\begin{array}{l}\text { Burned, } \\
\text { steam bath }\end{array}$ & & & \\
\hline $\begin{array}{l}\text { aSorghum bicolor } \\
\text { (L.) Moench } \\
\text { (Poaceae, MARE 15354) }\end{array}$ & $\begin{array}{l}\text { Süpürge } \\
\text { bitkisi }\end{array}$ & Fruits & Diarrhea & $\begin{array}{l}\text { Roasted, } \\
\text { eaten }\end{array}$ & & & \\
\hline $\begin{array}{l}\text { Teucrium polium L. } \\
\text { (Lamiaceae, MARE 15338) }\end{array}$ & Murad & $\begin{array}{l}\text { Aerial parts } \\
\text { Aerial parts } \\
\text { Aerial parts }\end{array}$ & $\begin{array}{c}\text { Stomach diseases } \\
\text { Appetizer } \\
\text { Diabetes }\end{array}$ & $\begin{array}{l}\text { Infusion, int. } \\
\text { Infusion, int. } \\
\text { Infusion, int. }\end{array}$ & & & $\begin{array}{c}\text { Stomach } \\
\text { diseases }(15,2) \\
\text { Diabetes }(2,6,8,14,16)\end{array}$ \\
\hline $\begin{array}{l}\text { Thymbra spicata L. } \\
\text { var. spicata } \\
\text { (Lamiaceae, MARE 15331) }\end{array}$ & Zahter & $\begin{array}{l}\text { Aerial parts } \\
\text { Aerial parts } \\
\text { Aerial parts }\end{array}$ & $\begin{array}{l}\text { Stomach diseases } \\
\text { Appetizer } \\
\text { Cold }\end{array}$ & $\begin{array}{l}\text { Infusion, int. } \\
\text { Infusion, int. } \\
\text { Infusion, int. }\end{array}$ & & & $(2)^{b}$ \\
\hline $\begin{array}{l}\text { Tribulus terrestris L. } \\
\text { (Zygophyllaceae, } \\
\text { MARE 15339) }\end{array}$ & Pitrak & Aerial parts & Kidney stones & Decoction, int. & & & $(2,11,12,14)^{b}$ \\
\hline $\begin{array}{l}\text { Verbascum sp. } \\
\text { (Scrophulariaceae, } \\
\text { MARE 15340) }\end{array}$ & Zarmasi & $\begin{array}{l}\text { Flowers \& leaves } \\
\text { Flowers \& leaves } \\
\text { Flowers \& leaves }\end{array}$ & $\begin{array}{l}\text { Wound } \\
\text { Sore throat } \\
\text { Cough }\end{array}$ & $\begin{array}{l}\text { Crushed, ext. } \\
\text { Infusion, int. } \\
\text { Infusion, int. }\end{array}$ & & & \\
\hline $\begin{array}{l}\text { Vitex pseudo-negundo } \\
\text { (Hausskn. ex Bornm.) } \\
\text { Hand.-Mazz } \\
\text { [Vitex agnus-castus } \\
\text { L. -Lamiacaeae ] } \\
\text { (Verbenaceae, } \\
\text { MARE 15330) }\end{array}$ & $\begin{array}{l}\text { Süpürge } \\
\text { bitkisi }\end{array}$ & $\begin{array}{l}\text { Flowering } \\
\text { branches }\end{array}$ & Cold & Decoction, int. & & & \\
\hline $\begin{array}{l}\text { Zea mays } \\
\text { L. subsp. mays } \\
\text { (Poaceae, MARE 15349) }\end{array}$ & Darı & Stylus & Kidney stones & Decoction, int. & $\begin{array}{l}\text { Fruit } \\
\text { Fruit }\end{array}$ & $\begin{array}{c}\text { Boiled/ } \\
\text { Roasted } \\
\text { Grinded for } \\
\text { making flour } \\
\text { [“mısır unu"] }\end{array}$ & $\begin{array}{l}\text { Urinary system } \\
\text { diseases (3) } \\
(10)^{\mathrm{b}}\end{array}$ \\
\hline
\end{tabular}

Int.: Internal use; Ext.: External use; ${ }^{\mathrm{C} C u l t i v a t e d ~ p l a n t ; ~}{ }^{\mathrm{D} D i f f e r e n t ~ u s a g e ~-~ D i r e c t l y ~ u s a g e ; ~(1) ~ A k a n ~ e t ~ a l . ~ 2008 ; ~(2) ~ A l t u n d a g ̆ ~ a n d ~ O ̈ z t u ̈ r k ~ 2011 ; ~(3) ~ B u l u t ~ e t ~ a l . ~ 2016 ; ~}$ (4) Çakılcıoğlu and Türkoğlu 2010; (5) Çakılcıoğlu et al. 2010; (6) Çakılcıoğlu et al. 2011; (7) Doğan and Tuzlacı 2015; (8) Mükemre et al. 2015; (9) Özgen et al. 2004; (10) Özgökçe and Özçelik 2004; (11) Sezik et al. 1997; (12) Șığva and Seçmen 2009; (13) Tabata et al. 1994; (14) Tetik et al. 2013; (15) Tuzlacı and Doğan 2010; (16) Yeșil and Akalın 2009 


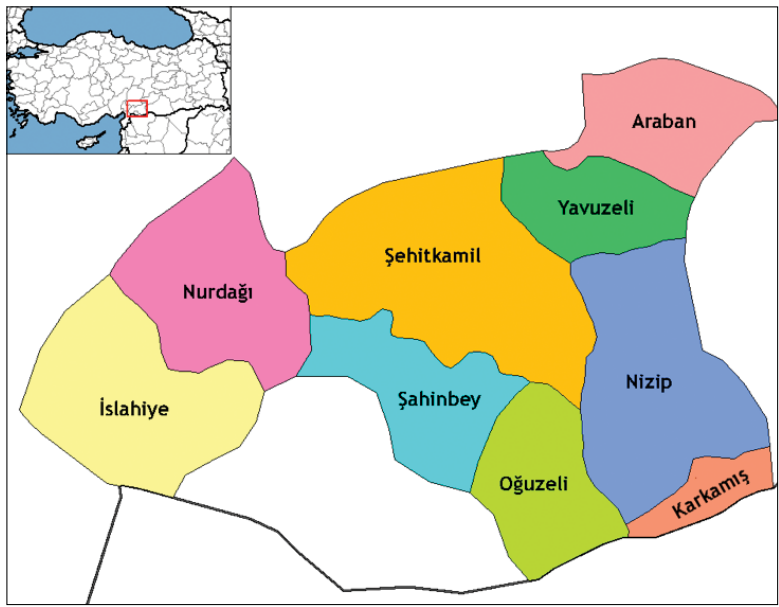

Figure 1. Map of Gaziantep (www.türkiyerehberi.com)

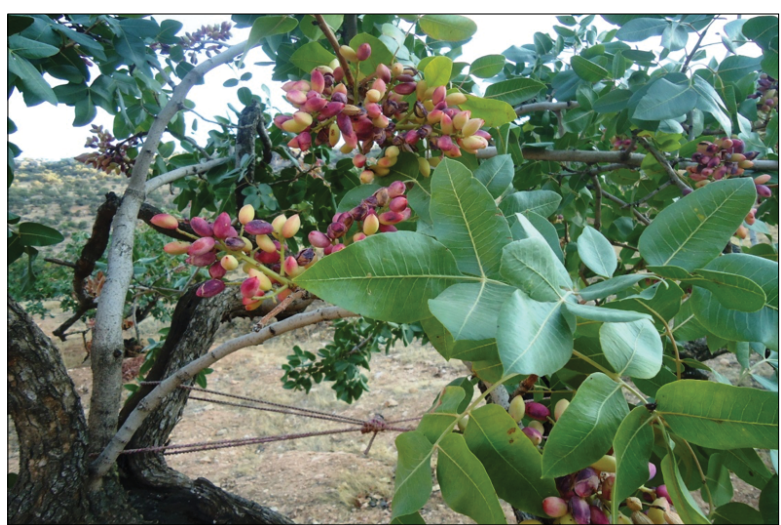

Figure 2. Pistacia vera $\mathrm{L}$.

\section{RESULTS AND DISCUSSION}

The plants used for medicinal purposes in Nizip are presented in Table 1. Taxonomical changes according to the plant list (http://www.theplantlist.org) were shown in parenthesis in Table 1 together with the popular scientific names. Twenty-seven medicinal plant species, belonging to 11 families, were recorded in the research area. Of these, 20 taxa were wild, and 7 taxa were cultivated plants (Figure 2). The most common usages of the plants were found to be gastrointestinal, respiratory system diseases and diabetes.

The main preparation methods was decoction and usually aerial parts were used in the preparation.

We compared our results with other comprehensive ethnobotanical studies on folk medicinal plants which have already been carried out in the neighbouring areas (Akan et al. 2008; Altundağ and Öztürk 2011; Bulut et al. 2016; Çakılcıoğlu and Türkoğlu 2010; Çakılcıoğlu et al. 2010, 2011; Mükemre et al. 2015; Özgen et al. 2004; Özgökçe and Özçelik 2004; Sezik et al. 1997; Şığva and Seçmen 2009; Tabata et al. 1994; Tetik et al. 2013; Tuzlacı and Doğan 2010; Yeşil and Akalın 2009) and presented in Table 1. Among them, Juglans regia L., Mentha Iongifolia (L.) Hudson, Rubus sanctus Schreber and Teucrium polium L. recorded in eight localities were the most commonly used herbal medicinal plants in Nizip and its surroundings.
Thirteen taxa used medicinally (Ficus carica L. subsp. carica, Hibiscus esculentus L., Juglans regia L., Mentha longifolia (L.) Hudson subsp. typhoides (Brig.) Harley, Olea europaea L. var. europaea, Pistacia vera L., Portulaca oleracea L., Punica granatum L., Quercus brantii Lindley, Rhus coriaria L., Rubus sanctus Schreber, Thymbra spicata L. var. spicata and Zea mays L. subsp. mays) were also used as food plants.

\section{Acknowledgements}

The authors wish to thank all the informants who contributed to this study with their knowledge and friendship.

\section{REFERENCES}

Akan H, Korkut MM, Balos MM (2008). Arat Dağı ve çevresinde (Birecik, Şanlıurfa) etnobotanik bir araştırma. Fırat Üniversitesi Fen ve Mühendislik Bilimleri Dergisi 20: 67-81.

- $\quad$ Alexiades MN (1996). Selected Guidelines for Ethnobotanical Research: A Field Manual. New York.

Altundağ E, Öztürk M (2011). Ethnomedicinal studies on the plant resources of East Anatolia, Turkey. Procedia Social and Behavioral Sciences 19: 756-777. [CrossRef]

- Bulut G, Tuzlacı E (2013). An ethnobotanical study of medicinal plants in Turgutlu (Manisa-Turkey). J Ethnopharmacol 149: 633647. [CrossRef]

Bulut G, Biçer M, Tuzlacı E (2016). The folk medicinal plants of Yüksekova (Hakkari-Turkey). J Fac Pharm Istanbul 46: 115-124.

Bulut G (2016). Medicinal and wild food plants of Marmara Island (Balikesir - Turkey). Acta Soc Bot Po/ 85: 1-16. [CrossRef]

- Cotton CM (1996). Ethnobotany: Principles and Applications. John Wiley and sons Ltd. West Sussex, UK.

Çakılcıoğlu U, Türkoğlu I (2010). An ethnobotanical survey of medicinal plants in Sivrice (Elazığ-Turkey). J Ethnopharmacol 132 : 165-175. [CrossRef]

- C Çakılcıoğlu U, Şengun MT, Türkoğlu I (2010). An ethnobotanical survey of medicinal plants of Yazıkonak and Yurtbaşı districts of Elazığ province, Turkey. J Med Plant Res 4: 567-572.

- Çakılcıoğlu U, Khatun S, Türkoğlu I, Hayta Ş (2011). Ethnopharmacological survey of medicinal plants in Maden (Elazığ-Turkey). J Ethnopharmaco/ 137: 469-486. [CrossRef]

- Davis PH (1965-1985). The Flora of Turkey and the East Aegean Islands. Vol. 1-9, Edinburgh University Press, Edinburgh.

- Davis PH, Mill RR, Tan K (1988). The Flora of Turkey and the East Aegean Islands. Vol. 10, Edinburgh University Press, Edinburgh. Doğan A, Tuzlaci E (2015). Wild edible plants of Pertek (Tunceli-Turkey). Marmara Pharm J 19: 126-135. [CrossRef]

Güner A, Özhatay N, Ekim T, Başer KHC (2000). The Flora of Turkey and the East Aegean Islands. Vol. 11, Edinburgh University Press, Edinburgh.

- Kaval I, Behçet L, Çakılcıoğlu U (2014). Ethnobotanical study on medicinal plants in Geçitli and its surrounding (Hakkari-Turkey). J Ethnopharmaco/ 155: 171-184. [CrossRef]

- Martin GJ (1995). Ethnobotany: A Methods Manual. Chapman and Hall, London. [CrossRef]

- Mükemre M, Behçet L, Çakılcıoğlu U (2015). Ethnobotanical study on medicinal plants in villages Çatak (Van-Turkey). J Ethnopharmacol 166: 361-374. [CrossRef]

Özgen U, Kaya Y, Coskun M (2004.) Ethnobotanical studies in the villages of the district of llıca (Province Erzurum) Turkey. Econ Bot 58: 691-696. [CrossRef]

- $\quad$ Özgökçe F, ÖzçelikH (2004). Ethnobotanical aspects of some taxa in East Anatolia (Turkey). Econ Bot 58: 697-704. [CrossRef]

- Sezik E, Yesilada E, Tabata M, Honda G, Takaishi Y, Tetsuro F, Tanaka T, Takeda Y (1997). Traditional Folk Medicine in Turkey VIII. Folk Medicine in East Anatolia; Erzurum, Erzincan, Ağrı, Kars, Iğdır Provinces. Econ Bot 51: 195-211. [CrossRef] 
- S Şı̆gva HÖ, Semen Ö (2009). Ethnobotanical survey of |şıklı (Çarpın), Dağdancık and Tokdemir in Gaziantep, Turkey. IUFS J Biol 68: $19-26$.

- Tabata M, Sezik E, Honda G, Yesilada E, Fukui H, Goto K, Ikeshiro Y (1994). Traditional Medicine in Turkey III. Folk Medicine in East Anatolia, Van and Bitlis Provinces. Pharmaceutical Biology 32 : 3-12. [CrossRef]
Tetik F, Civelek \$̧, Çakılcıoğlu U (2013). Traditional uses of some medicinal plants in Malatya (Turkey). J Ethnopharmacol 146: 331-346. [CrossRef]

- Tuzlacı E, Doğan A (2010). Turkish folk medicinal plants, IX: Ovacik (Tunceli). Marmara Pharm J 14: 136-143. [CrossRef]

Yeşil Y, Akalın E (2009). Folk medicinal plants in Kürecik area (Akçadağ/Malatya-Turkey). TurkJ Pharm Sci 6: 207-220. 
Istanbul J Pharm 47 (2): 57-62

\section{Appendix 1}

\section{Questionnaire Form}

1. Name and surname of the participant

2. Age and sex of the participant

3. Telephone and address of the participant

4. Educational level of the participant

5. Date of interview

6. Place of residence of the participant

7. Duration of residence of the participant

8. Local name of the plant

9. Human health or animal health

10. Ailments treated/therapeutic effect

11. Plant part used

12. Preparation

13. Administration

14. Dosage

15. Duration of treatment

16 Age group of patients (baby, children, adults)

17. Side effect

18. Different ethnobotanical use 